\title{
Estudos de Síntese do Sulfureto de Zinco e Efeitos Quânticos de Dimensão
}

\author{
A N A A TARINA C. ESTEVES, TITO TRINDADE*
}

\section{Introdução}

\subsection{Contexto pedagógico e objectivos}

Apresenta-se um caso de estudo na área da Química Inorgânica e de Materiais com recurso à realizaçâo de trabaIho laboratorial, nomeadamente a técnicas de caracterização frequentemente utilizadas pelo Químico. Este caso de estudo foiapresentado a alunos de mestrado após terem frequentado aulas sobre técnicas de caracterização de materiais leccionadas por investigadores especialistas nessas técnicas. Neste trabalho, estas técnicas foram integradas em componentes experimentais realizadas por diferentes grupos de alunos, com formações de base distintas, mas pertencentes a uma só equipa de investigação. Optou-se por distinguir a aplicabilidade das técnicas a situações práticas com graus de dificuldade distintos. A opção por esta metodologia justifica-se essencialmente por duas situações indissociáveis: 1) o trabalho foi apresentado no âmbito da disciplina de Caracterização Química do curso de Mestrado em Ciência e Engenharia de Materiais, frequentado por alunos com preparação química laboratorial muito diversa; II) possibilidade de fomentar o trabalho de equipa inter-disciplinar, recorrendo às competências diversas dos alunos que decorrem da sua própria formação de licenciatura. 0 trabalho pode ser facilmente adaptado a outras situações menos atípicas, por exemplo, quando se verifique uma maior uniformidade na formação de base dos alunos. Sugere-se no entanto que este trabalho seja dirigido a formandos que frequentem um curso de pós-graduação ou os últimos anos da sua formação universitária inicial. O tempo para a realização do trabalho experimental, com distribuição de tarefas pelos vários grupos de alunos, foi de 6 horas. 0 relatório global de trabaIho com recurso a pesquisa bibliográfica foi entregue 2 semanas depois da aula laboratorial.

Neste caso de estudo pretende-se integrar um conjunto de conceitos actuais ao nível da síntese química e caracterização de materiais. Na primeira parte do trabalho é proposta a preparação de pós de um semicondutor de importância tecnológica actual, o sulfureto de zinco, recorrendo ao método dos precursores moleculares e à precipitação directa em solução aquosa. A segunda parte do trabalho laboratorial consiste na preparação de nanopartículas de ZnS, cujas propriedades ópticas poderão ser comparadas com as propriedades do ZnS macrocristalino.

Os objectivos específicos do trabalho são:

- Saber utilizar e adequar a um caso de estudo, técnicas de caracterização de compostos inorgânicos, nomeadamente métodos espectroscópicos, análises térmicas e difracção de raios $X$ do pó.

- Reconhecer a importância de diferentes métodos de síntese na preparação de materiais com propriedades distintas.
- Interpretar as propriedades ópticas de nanopartículas semicondutoras (ZnS) tendo em conta efeitos quânticos de dimensão.

Segue-se um resumo dos tópicos que os alunos deveriam abordar no relatório escrito, contextualizando cientificamente o trabalho prático realizado. Ter-se adoptado como estratégia de trabalho a formação de uma equipa de investigação pluri-disciplinar, permitiu que fossem abordados por intermédio da discussão crítica, conceitos fundamentais de Química, e de algum modo também de Física e Ciência dos Materiais, que se supunha estarem presentes de um modo diferenciado

\subsection{Preparação de sólidos a partir de precursores moleculares}

O recurso a compostos químicos de natureza molecular para preparar materiais é um processo utilizado já há algum tempo, nomeadamente na preparação de materiais cerâmicos [1,2]. Refira-se, por exemplo, a preparação de óxidos metálicos de pureza elevada pela calcinação dos respectivos oxalatos ou acetilacetonatos. Foi no entanto nas últimas décadas do século passado que se verificou um interesse acentuado pela preparação de materiais recorrendo ao design de precursores moleculares novos.[3-8] Esta situação, em particular, reflecte uma mudança fundamental ao nível da preparação de materiais, na medida em que pressupõe que as propriedades finais do material a produzir podem ser desde logo condicionadas na 


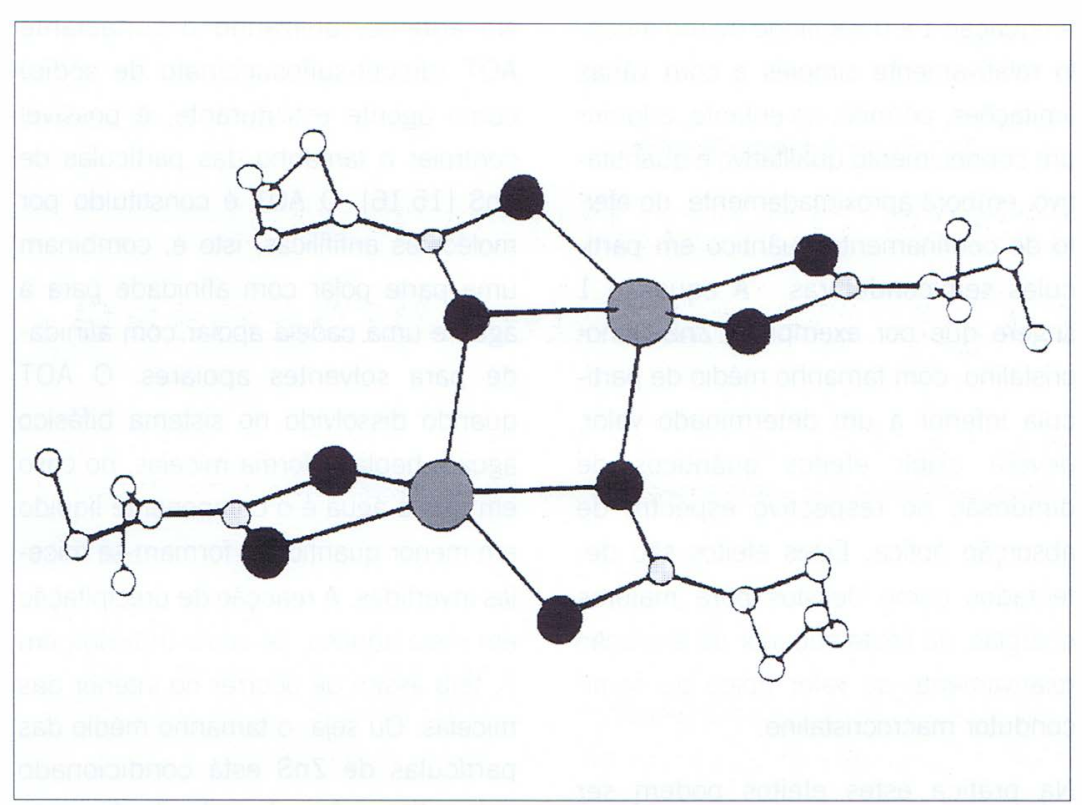

figura 1 Estrutura molecular do composto $\left[\mathrm{Zn}\left\{\mathrm{S}_{2} \mathrm{CN}\left(\mathrm{C}_{2} \mathrm{H}_{5}\right)_{2}\right\}_{2}\right]_{2}[7]$.

fase da síntese química. A investigação química neste domínio tem sido notável, permitindo não só optimizar processos tradicionais de preparação de materiais bem como levar ao aparecimento de novos produtos

O trabalho proposto envolve a preparação do semicondutor ZnS por decomposição térmica do complexo dimérico $\left[\mathrm{Zn}\left\{\mathrm{S}_{2} \mathrm{CN}\left(\mathrm{C}_{2} \mathrm{H}_{5}\right)_{2}\right\}_{2}\right]_{2}: \mathrm{Zn}\left(\mathrm{Et}_{2} \mathrm{DTC}\right)_{2}$ (Figura 1). As moléculas deste precursor têm a particularidade de conter quimicamente ligados os elementos que constituem o semicondutor final, neste caso, o zinco e o enxofre. Por esta razão, designam-se estes precursores por unimoleculares (single-molecule precursors) para os distinguir dos precursores moleculares convencionais, em que os elementos constituintes do material final são obtidos a partir de compostos distintos. O composto $\mathrm{Zn}\left(\mathrm{Et}_{2} \mathrm{DTC}\right)_{2}$ será caracterizado recorrendo a técnicas instrumentais frequentemente utilizadas pelo químico, como sejam a espectroscopia de absorção no infravermeiho (IV) e a espectroscopia de ressonância magnética nuclear (RMN). Uma vez que se pretende obter o ZnS por decomposição térmica do $\mathrm{Zn}\left(\mathrm{Et}_{2} \mathrm{DTC}\right)_{2}$, torna-se relevante o estudo do comportamento térmico do precursor, nomeadamente por termogravimetria (TG).
O ZnS cristaliza com uma estrutura cúbica ( $\beta$-ZnS: esfalerite ou blenda de zinco) ou com uma estrutura hexagonal $(\alpha$-ZnS:wurtzite). [9,10] Ambas as estruturas baseiam-se em redes derivadas de um empacotamento compacto de aniões com metade dos interstícios tetraédricos ocupados, sendo portanto a geometria de coordenação dos iões tetraédrica e o número de coordenação 4:4 $[9,10]$. Trata-se de um semicondutor directo com um valor de $E_{\mathrm{g}}$ igual a 3,8 eV (fase hexagonal) ou 3,6 eV (fase cúbica), à temperatura ambiente. A fase hexagonal é obtida geralmente a temperatura elevada enquanto em condições ambiente a fase cúbica é mais estável. As condições experimentais utilizadas na preparação do ZnS podem no entanto condicionar o tipo de fase cristalina presente na amostra em condições ambiente [7].

O ZnS policristalino é um composto que no estado puro ou dopado tem aplicações tecnológicas diversas, distinguindo-se a sua utilização como pigmento luminescente em tubos de raios catódicos. O desempenho deste pigmento depende de propriedades físico-químicas, como por exemplo a pureza química, tipo de estrutura e grau de cristalinidade. Estas por sua vez dependem do método envolvido na preparação do ZnS. Em aplicações para opto-electrónica, o ZnS é um dos materiais investigados na deposição de hetero-estruturas epitaxiais, como por exemplo a representada na Figura 2. [11] Estas hetero-estruturas têm interesse para o fabrico de laser de semicondutores, um dispositivo utilizado nas impressoras laser ou nos leitores de CD. Os laser de semicondutores utilizados nestas aplicações operam na região do visível a maiores comprimentos de onda. O fabrico de um laser de semicondutor a operar na região do verde/azul possibilitaria a comunicação a longa distância por fibras ópticas submarinas ou o fabrico de dispositivos de memória com elevada

$1,5 \mu \mathrm{m} \quad \mathrm{n}-\mathrm{ZnS}_{x} \mathrm{Se}_{\mathrm{x}-1} \mathrm{Cl}$

$75 \AA / 100 \AA \overline{\text { Poço quântico }}$

$2,0 \mu \mathrm{m} \quad \mathrm{p}-\mathrm{ZnS}_{\mathrm{x}} \mathrm{Se}_{\mathrm{x}-1}: \mathrm{N}$

$1,5 \mu \mathrm{m}$ tampão de p-GaAs

substrato de p-GaAs

figura 2 Esquema de uma hetero-estrutura epitaxial [11]

densidade de informação. Os métodos de deposição química de hetero-estruturas contendo ZnS poderão desempenhar um papel relevante nesse sentido.

\subsection{Efeitos quânticos de dimensão em semicondutores nanocristalinos}

Nos últimos anos, houve um interesse grande na investigação em nanomateriais, nomeadamente pelo impacto tecnológico que esse tipo de materiais terá num futuro próximo. Um dos aspectos mais interessantes neste tipo de sólidos, é o facto de apresentarem propriedades físicas distintas dos materiais macrocris- 
talinos análogos, devido a um efeito de dimensão de partícula $[12,13]$. A dimensão de nanocristais é intermédia entre as dimensões típicas de moléculas e do composto macrocristalino (Figura 3). Investigação recente demonstrou que ocorrem alterações significativas nas propriedades ópticas, electrónicas e mecânicas de um material quando este passa da forma macrocristalina a um material constituído por partículas de dimensões nanométricas (1-10 nm) $[12,13]$. Neste trabalho focam-se aspectos relacionados com a síntese e propriedades ópticas de nanopartículas de ZnS, tornando-se evidente a importância do controlo do tamanho de partícula por via química ao nível das propriedades finais do ZnS. De facto, com a diminuição do tamanho médio de partícula de um semicondutor observa-se um aumento do hiato óptico $\left(E_{g}\right)$ (Figura 3).[12-14] Este comportamento é um exemplo de efeito quântico de dimensão num semicondutor. O aumento de Eg para partículas semicondutoras de dimensões nanométricas resulta do confinamento quântico tridimensional dos transportadores de carga, devido às dimensões das nanopartículas. Utilizando o modelo da partícula numa caixa, Brus deduziu a equação 1 que relaciona $\circ E_{g}$ do semicondutor nanocristalino com o tamanho de partícula $[12,14]$ :

$\Delta \mathrm{E} \cong \frac{\hbar^{2} \pi^{2}}{2 \mathrm{R}^{2}}\left[\frac{1}{\mathrm{~m}_{\mathrm{e}}{ }^{*}}+\frac{1}{\mathrm{~m}_{\mathrm{h}}}\right]-\frac{1.8 \mathrm{e}^{2}}{\varepsilon \mathrm{R}}$

(1)

em que

$\Delta \mathrm{E}=$ desvio relativamente ao valor de $\mathrm{E}_{\mathrm{g}}$ do material macrocristalino

$\hbar=$ constante de Planck

$\mathrm{R}=$ dimensão característica do nanocristal (raio)

$\mathrm{m}_{\mathrm{e}}{ }^{*}=$ massa efectiva do electrão;

$m_{n}{ }^{*}=$ massa efectiva da lacuna

$\mathrm{e}=$ carga elementar

$\varepsilon=$ permitividade
A equação 1 é o resultado de um modelo relativamente simples e com várias limitações, permite, no entanto, adquirir um conhecimento qualitativo e quantitativo, embora aproximadamente, do efeito de confinamento quântico em partículas semicondutoras. A equação 1 sugere que por exemplo o ZnS nanocristalino, com tamanho médio de partícula inferior a um determinado valor, deverá exibir efeitos quânticos de dimensão no respectivo espectro de absorção óptica. Estes efeitos são detectados como desvios para maiores energias, do limite superior de absorção relativamente ao valor típico do semicondutor macrocristalino.

$\mathrm{Na}$ prática estes efeitos podem ser demonstrados preparando $\mathrm{ZnS}$ de dimensões nanométricas por um método de precipitação na presença de sistemas anfifílicos. Para efeitos de comparação será preparado primeiramente ZnS em pó, com propriedades morfológicas não controladas, pela simples mistura de soluções aquosas dos sais $\mathrm{Zn}\left(\mathrm{NO}_{3}\right)_{2}$ e $\mathrm{Na}_{2} \mathrm{~S}$ :

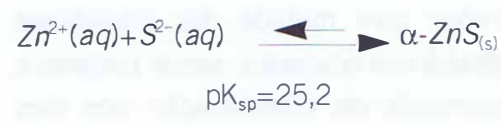

(2)
No entanto, utilizando o surfactante AOT (dioctil-sulfosuccinato de sódio) como agente estruturante, é possivel controlar o tamanho das partículas de ZnS $[15,16]$. O AOT é constituído por moléculas anfifilicas, isto é, combinam uma parte polar com afinidade para a água e uma cadeia apolar com afinidade para solventes apolares. O AOT quando dissolvido no sistema bifásico água/n-heptano forma micelas, no caso em que a água é o componente líquido em menor quantidade formam-se micelas invertidas. A reacção de precipitação em meio aquoso, tal como indicada em 2, terá assim de ocorrer no interior das micelas. Ou seja, o tamanho médio das partículas de ZnS está condicionado pelas dimensões da gota de água no interior das micelas $[15,16]$, podendo esta ser controlada pela razão $\mathrm{R}$ :

$$
\mathrm{R}=[\text { água }] /[\mathrm{AOT}]
$$

\section{Experimental}

Os pontos 2.1 a 2.3 referem-se à parte experimental deste trabalho e estão disponiveis na versão on-line da Química em http://www.spq.pt:

\subsection{Normas de segurança específicas}

2.2. Caracterização de $\mathrm{ZnS}$ macrocristalino sintetizado por decomposição térmica de um precursor uni-molecular

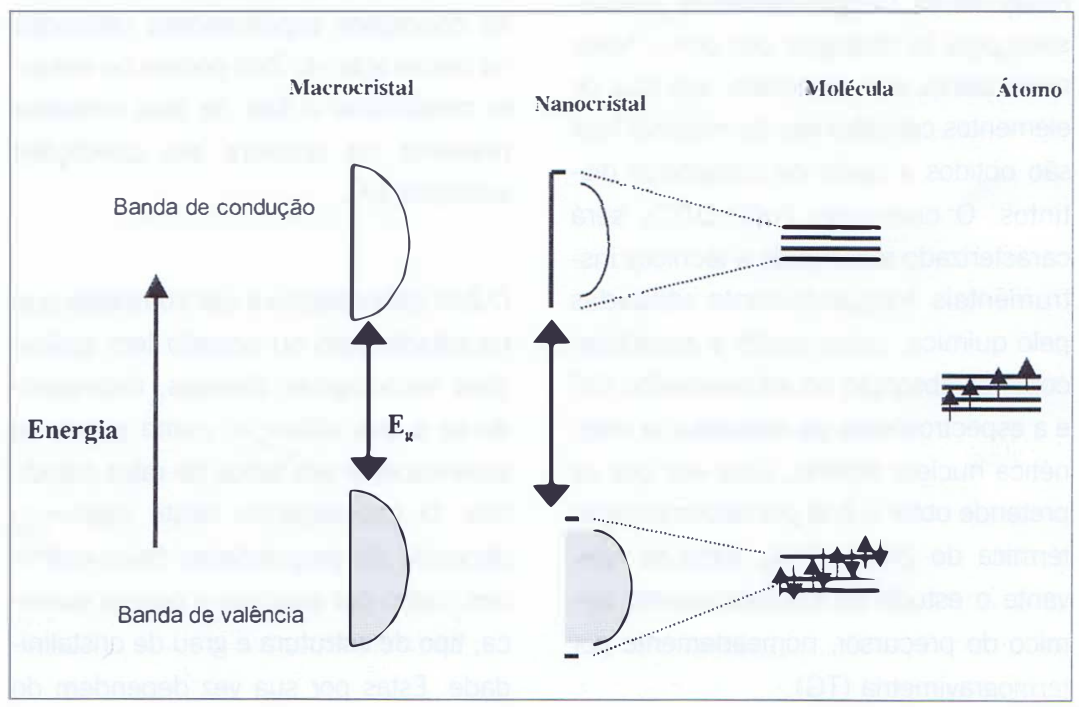


2.2.1 Síntese do complexo dietiloditiocarbamato de zinco: $\mathrm{Zn}\left(\mathrm{Et}_{2} \mathrm{DTC}\right)_{2}$

\subsubsection{Caracterização do composto $\mathrm{Zn}\left(\mathrm{Et}_{2} \mathrm{DTC}\right)_{2}$}

2.2.3 Caracterização de ZnS macrocristalino obtido a partir de $\mathrm{Zn}\left(\mathrm{Et}_{2} \mathrm{DTC}\right)_{2}$

\subsection{Preparação e caracterização de}

ZnS nanocristalino: estudo de efeitos quânticos de dimensão de massa obtida e estequiométrica. Critique o resultado obtido.

4. O que entende por polimorfismo? Comente a importância da técnica de difracção de raios $X$ do pó no contexto deste trabalho.

5. Utilizando o espectro de reflectância no visível do ZnS macrocristalino, faça uma estimativa do valor do hiato óptico deste semicondutor, à temperatura am. biente. dados espectroscópicos e a análise térmica efectuada. Na Tabela 1 resumem-se os resultados pertinentes.

Os picos no espectro de RMN do Zn $\left(\mathrm{Et}_{2} \mathrm{DTC}\right)_{2}$ foram atribuídos aos grupos etilo do composto. $\mathrm{O}$ espectro de infravermelho do composto $\mathrm{Zn}\left(\mathrm{Et}_{2} \mathrm{DTC}\right)_{2}$ evidencia uma banda forte a $1498 \mathrm{~cm}^{-1}$, sugerindo um caracter intermédio de ligação dupla e polar da ligação $\mathrm{C}-\mathrm{N}$ [17]. O composto $\mathrm{Zn}\left(\mathrm{Et}_{2} \mathrm{DTC}\right)_{2}$ apresen-

tabela 1

\begin{tabular}{|ccc|}
\hline${ }^{1} \mathrm{H} \mathrm{RMN}\left(\mathrm{CDCl}_{3}+\mathrm{TMS}\right)$ & IV & Termogravimetria \\
\hline $1,27 \mathrm{ppm} ; 3 \mathrm{H}$; tripleto: $\left(\mathrm{C}_{3}\right)$ & $1498 \mathrm{~cm}^{-1} \mathrm{v}(\mathrm{C}-\mathrm{-N})$ & $\Delta \mathrm{m}_{\text {estequiométrica }}=73 \%$ \\
\hline $3,81 \mathrm{ppm} ; 2 \mathrm{H}$; quadrupleto: $\left(\mathrm{C}_{\mathrm{H}} .2\right)$ & $994 \mathrm{~cm}^{-1} \mathrm{v}(\mathrm{C}-\mathrm{S})$ & $\Delta \mathrm{m}_{\text {observada }}=81 \%$ \\
\hline & & $\Delta \mathrm{T}=316-362{ }^{\circ} \mathrm{C}$ \\
\hline
\end{tabular}

2.3.1 Preparação de pós de ZnS por precipitação directa em solução aquosa.

2.3.2 Preparação de nanopartículas de $\mathrm{ZnS}$

2.3.3 Estudo de efeitos quânticos de tamanho no espectro óptico do ZnS

\section{Tratamento e interpretação dos resultados experimentais.}

Foram colocadas aos mestrandos questões que Ihes permitissem reflectir sobre os resultados obtidos durante a realização do relatório do trabalho prático. Exemplificam-se algumas dessas questões.

1. Identifique no espectro de infravermelho do $\mathrm{Zn}\left(\mathrm{Et}_{2} \mathrm{DTC}\right)_{2}$ as bandas carac terísticas dos modos de elongação dos grupos $\mathrm{C}=\mathrm{S}$ e $\mathrm{C}=\mathrm{N}$. Comente os resultados obtidos.

2. Interprete o espectro de RMN de protão do composto $\mathrm{Zn}\left(\mathrm{Et}_{2} \mathrm{DTC}\right)_{2}$.

3. Com base no termograma do composto $\mathrm{Zn}\left(\mathrm{Et}_{2} \mathrm{DTC}\right)_{2}$ proponha um esquema químico para a reacção de decomposição térmica. Indique as variações
6. Tendo em conta a equação de Brus e o valor de $E_{g}$ obtido para o pó de ZnS, interprete os espectros do visível do ZnS coloidal.

Apresentam-se seguidamente alguns dos resultados mais relevantes obtidos pelos mestrandos e respectivos comentários.

$\mathrm{O} \mathrm{Zn}\left(\mathrm{Et}_{2} \mathrm{DTC}\right)_{2}$ de zinco utilizado como precursor molecular na preparação de ZnS foi identificado tendo em conta

figura 4 Padrōes de difracção de raios $\mathrm{X}$ do pó de $\mathrm{ZnS}$ obtido por : a)por decomposição térmica do $\mathrm{Zn}\left(\mathrm{Et} \mathrm{t}_{2} \mathrm{DTC}\right)_{2}$ e b) precipitaçāo.

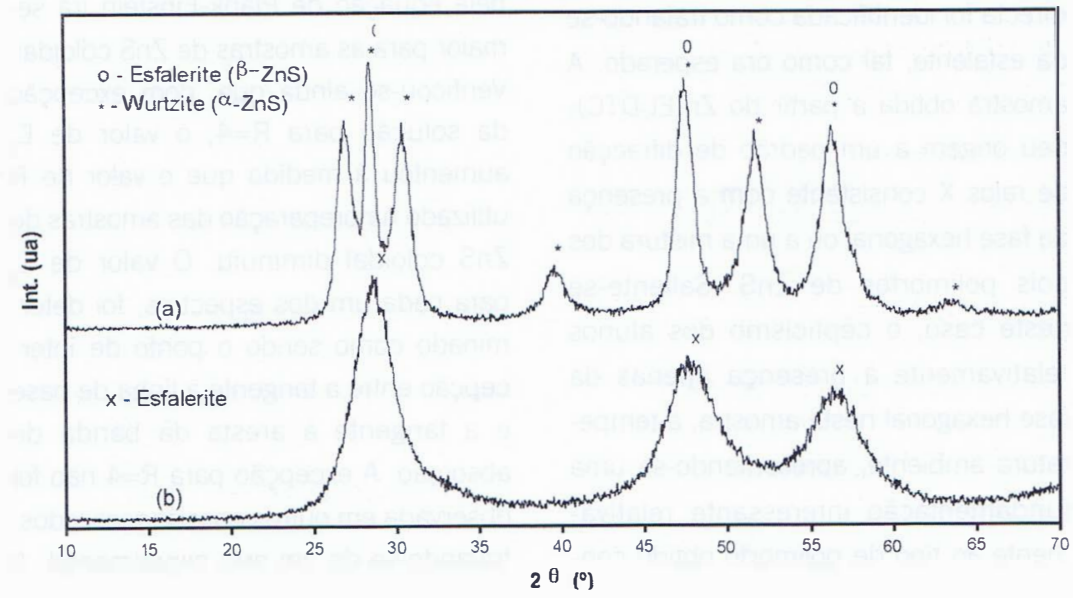

ta uma banda forte a $994 \mathrm{~cm}^{-1}$ atribuída ao modo $v$ (CS), confirmando que o composto $\mathrm{Zn}\left(\mathrm{Et}_{2} \mathrm{DTC}\right)_{2}$ é um quelato, por oposição à forma éster que deveria originar duas bandas à volta de 1000 $\mathrm{cm}^{-1}$.[17] Foi identificada uma banda a $379 \mathrm{~cm}^{-1}$ atribuída ao modo $v(\mathrm{Zn}-\mathrm{S})$, confirmando a coordenação do ditiocarbamato ao catião zinco. Os resultados obtidos por termogravimetria concordam razoavelmente com a decomposição térmica num único passo, do 


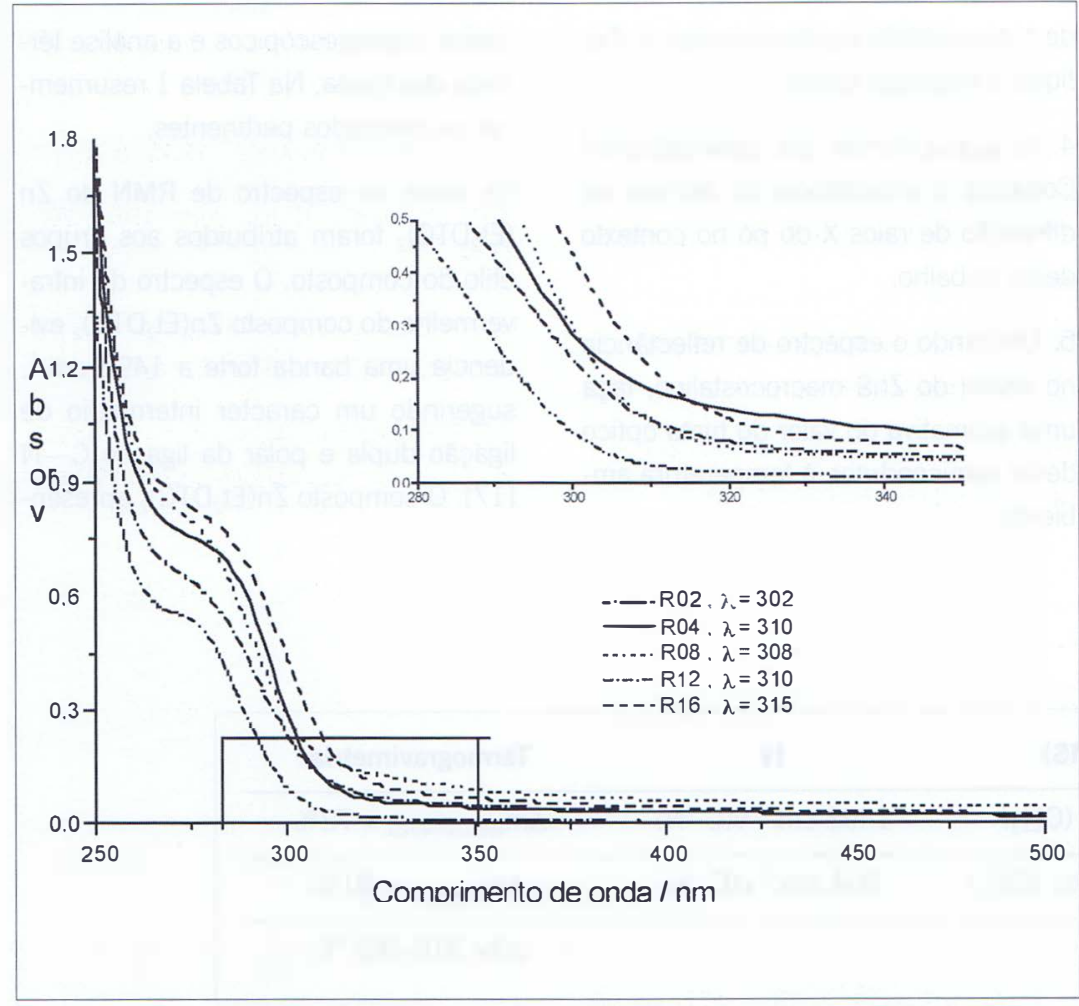

$\mathrm{Zn}\left(\mathrm{Et}_{2} \mathrm{DTC}_{2}\right.$ no sólido ZnS. O ZnS obtido após calcinação pode apresentar uma coloração acinzentada devido a carbono residual presente na amostra. Este facto permite que os alunos critiquem o método utilizado na preparação do semicondutor e sugiram soluções que permitam eliminar este tipo de contaminação do ZnS [7,8].

As amostras de ZnS macrocristalino obtidas por decomposição térmica do $\mathrm{Zn}\left(\mathrm{Et}_{2} \mathrm{DTC}\right)_{2}$ e pelo método de precipitação directa foram caracterizadas por difracção de raios $X$ do pó (Figura 4). A amostra de ZnS obtida por precipitação directa foi identificada como tratando-se da esfalerite, tal como era esperado. A amostra obtida a partir do $\mathrm{Zn}\left(\mathrm{Et}_{2} \mathrm{DTC}\right)_{2}$ deu origem a um padrão de difracção de raios $X$ consistente com a presença da fase hexagonal ou a uma mistura dos dois polimorfos de ZnS. Saliente-se neste caso, o cepticismo dos alunos relativamente à presença apenas da fase hexagonal nesta amostra, à temperatura ambiente, apresentando-se uma fundamentação interessante relativamente ao tipo de polimorfo obtido con- soante os parâmetros experimentais envolvidos [7].

A Figura 5 mostra os espectros UV/vis para os colóides de $\mathrm{ZnS}$ nanocristalino. A comparação dos valores de $\lambda_{\text {onset }}$ do ZnS nanocristalino obtido para diferentes valores de $R$, com o $\lambda_{\text {onset }}=370 \mathrm{~nm}$ no espectro de reflectância difusa do ZnS obtido por precipitação directa, levou os alunos a concluirem que o $\mathrm{ZnS}$ nanocristalino exibe efeitos quânticos de dimensão no espectro óptico. De facto, o $\lambda_{\text {onset }}$ das amostras de $\mathrm{ZnS}$ coloidal encontra-se desviado para menores valores relativamente ao $\lambda_{\text {onset }}$ do $\mathrm{ZnS}$ em pó, consequentemente, o $E_{g}$ obtido pela equação de Plank-Einstein irá ser maior para as amostras de ZnS coloidal. Verificou-se ainda que, com excepção da solução para $R=4$, o valor de $E_{g}$ aumentou à medida que $\mathrm{o}$ valor de $\mathrm{R}$ utilizado na preparação das amostras de ZnS coloidal diminuiu. $O$ valor de $E_{8}$ para cada um dos espectros, foi determinado como sendo o ponto de intercepção entre a tangente à linha de base e a tangente à aresta da banda de absorção. $A$ excepção para $R=4$ não foi observada em outros ensaios realizados, tratando-se de um erro experimental. A figura 5 espectros UV/vis para os colóides de ZnS nanocristalino

variação de $E_{g}$ com o valor de $R$, e por conseguinte com o tamanho médio de partícula, foi explicada tendo em conta efeitos quânticos de dimensão no ZnS nanocristalino, tal como sugerido pela Figura 3. A aplicação da equação de Brus permitiu que os alunos confirmassem quantitativamente esta interpretação, estimando as dimensões de partícula (tipicamente à volta de $3 \mathrm{~nm}$ de diâmetro) e utilizando os valores pertinentes obtidos da bibliografia $[14,15]$ para as constantes que figuram na equação de Brus.

\section{Conclusão}

O trabalho apresentado permitiu que alunos de mestrado com formação de licenciatura diversa, nomeadamente de Física, Química, Mecânica e Materiais, tomassem contacto com técnicas de síntese e caracterização de compostos inorgânicos. O tópico proposto como caso de estudo foi de interesse geral para os mestrandos nomeadamente pela sua actualidade. Os objectivos propostos para o trabalho foram de um modo geral atingidos, quer na componente de síntese química quer na com- 
ponente de caracterização. O trabalho experimental proposto pode ser facilmente adaptado à preparação de CdS em vez de ZnS [6,15]. Esta alteração implica cuidados acrescidos no manuseamento de soluções e compostos contendo cádmio, um metal tóxico. A utilização deste semicondutor apresenta, no entanto, a vantagem de ser mais fácil a monitorização das propriedades ópticas por espectroscopia no visivel, uma vez que tem o $E_{g}$ (CdS macrocristalino) igual a 2,4 eV. Refira-se que a natureza da superfície de materiais nanocristalinos não foi abordada neste caso de estudo. Dada a sua importância, o estudo das propriedades de superfície de nanomateriais é uma possibilidade de desenvolvimento futuro deste trabalho.

\section{Agradecimentos}

Agradece-se a contribuição prestada pelos alunos do Mestrado em Ciência e Engenharia de Materiais (Edição 2000) da Universidade de Aveiro.

A. C. C. Esteves agradece uma bolsa de investigação da Universidade de Aveiro.

Referências

[1] C. N. R. Rao, J. Gopalakrishnan, New Directions in Solid State Chemistry, Cambridge University Press.

[2] M. T. Weller, Inorganic Materials Chemistry, Oxford University Press, 1994, p.26.

[3] P. O'Brien, Precursors for Electronic Materials in Inorganic Materials, (ed. D. W. Bruce, D- O'Hare), John Wiley \& Sons, 1992, p. 491

[4] M. Bochmann, Chem. Vap. Deposition, $1996,2,85$

[5]D. Segal, Chemical Synthesis of Advanced Ceramic Materials-Chemistry of Solid State Materials, 1, A. R. West, H. Baxter (Eds), Cambridge University press, Great Britain, 1989.
[6] T. Trindade, P. O'Brien, X. Zhang, Chem. Mater. 1997, 9, 523

[7] R. D. Pike, H. Cui, R. Kershaw, K Dwight, A. Wold, T. N. Blanton, A. A. Wernberg, H. J. Gysling, Thin Solid Films, 1993, 224, 221.

[8] D. M. Frigo, O. F. Z. Khan, P. O’Brien, J. Crystal Growth, 1989, 96, 989.

[9] A. C. Cavaleiro, Química Inorgânica Básica, Universidade de Aveiro, 1997.

[10] D. F. Shriver, P. W. Atkins, Inorganic Chemistry, 3rd edition, Oxford University Press, 1999

[11] R. Gunshor, A. Nurmikko, M. Kobayashi, Physics Today, 49, March 1992.
[12] M. L. Steigerwald, L. E. Brus, Acc. Chem. Res., 1990, 23, 183.

[13] H. Weller, Adv. Mater., 1993, 5, 88 .

[14] L. E. Brus, J. Chem. Phys., 1984, 80, 4403.

[15] R. R. Chandler, S. R. Bigham, J. L. Coffer, J. Chem. Educ., 1993, 70, A7.

[16] T. F. Towey, A. Khan-Lodi, B. H. Robinson, J. Chem. Soc. Faraday Trans., 1990, 86, 3757.

[17] F. Bonati, R. Ugo, J. Organometal. Chem., 1967, 10, 257

\section{KSNIIK- TECH}

Kromatografia + EspeKtroscopia

CROMATOGRAFIA: HRGC / HPLC ESPECTROSCOPIA/ESPECTROMETRIA ENGENHARIA EQUIPAMENTOS DE LABORATÓRIO PREPARAÇÃO DE AMOSTRAS CIÊNCIA DE MATERIAIS/NÁCUO

Konik-Tech, S.A.

Rua Prof. Veiga Ferreira, 6B 1600 Lisboa

Telef. 217573547

Fax. 217573485

E-mail: lisboa@konik-group.com

Vendas: sales@konik-group.com Marketing: marketing@konik-group.com Serviço Técnico: SAT@konik-group.com www.konik-group.com 